\title{
Preparation of Liposomes for Negative Staining TEM
}

\author{
Ulrich Baxa
}

Frederick National Laboratory for Cancer Research, Frederick, Maryland, United States

With the strong increase of interest in cryo-electron microscopy the method of negative staining has also experienced a resurgence. Many use it as a quality control step for cryo-EM sample preparation, cryo-EM facilities often use it as a prerequisite when applying for imaging time, and negative staining can also reveal useful information in its own right. Compared to cryo-EM, negative staining is simple, quick, does not require any special instrumentation, and can be imaged even on lower level TEMs. All the preparation steps and imaging are performed at room temperature.

Negative staining is well established for proteins and most of the time proteins and protein complexes are preserved close to the native state [1]. Unfortunately the same is not always true for liposome and other lipid preparations, which are much more susceptible to artifacts in negative stain [2] (Figure 1 and 2). Results from negative staining of liposomes should be interpreted carefully and especially unexpected findings should be crosschecked with other methods, for example cryo-EM. Optimization of staining protocols are often needed to adapt to different liposome preparations. Once a preparation method has been validated for a particular liposome preparation it can be used to check concentration, size distribution and morphology (e.g. liposomes vs. bicelles) of similar preparations (Figure 1).

The main problem in negative staining of liposomes is that these can change morphology during the process. This is due to interaction of the lipids with the carbon surface and/or changes in ionic strength and osmolarity during the staining and air drying. Liposomes can change their shape and in extreme cases completely lose integrity and spread out onto the carbon film (Figure 2). At higher concentration this can lead to a continuous film of lipids over the complete grid preventing any useful observations. Often these problems can be resolved by diluting the liposome preparation and/or varying the standard negative stain procedure with the goal of reducing the time liposomes interact with carbon film and increasing the speed of drying. In the tutorial several variations of the negative stain protocol achieving this goal will be presented. Even in cases when liposomes stay intact they will flatten onto the carbon film during the drying process and appear larger than the original in solution (Figure 2) [3].
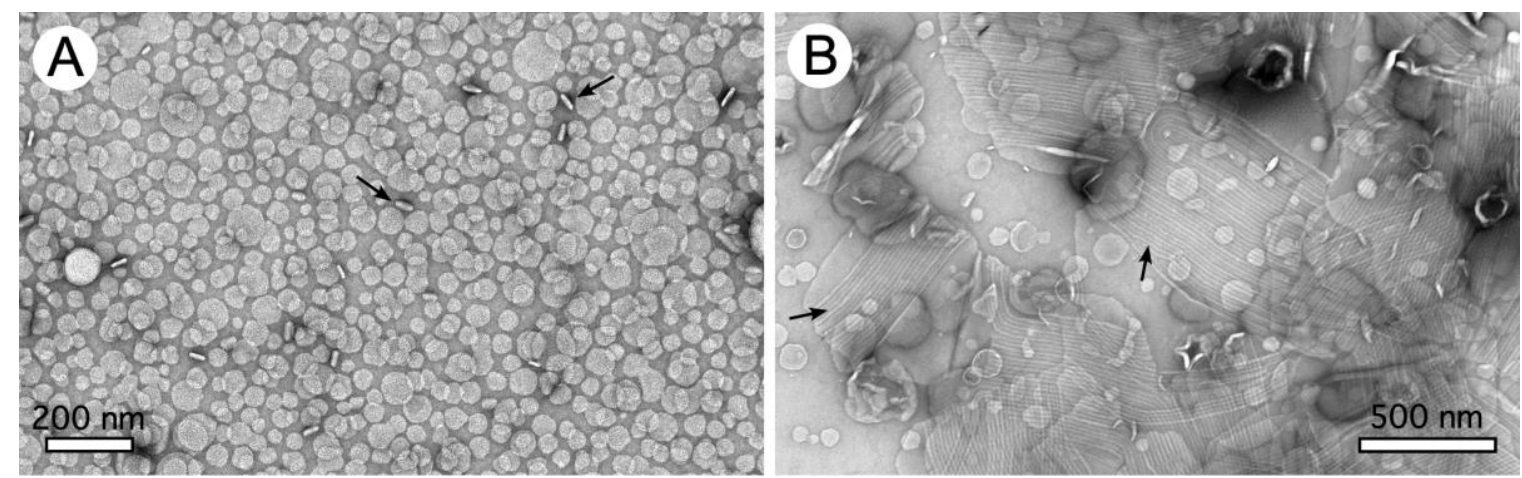

Figure 1. Examples of negatively stained liposome preparations. (A) Liposome preparation with a relatively monodisperse size distribution containing a few bicelles (arrows). In negative stain, bicelles can be recognized only when visualized on edge (side view). Top views of bicelles will appear very similar to liposomes. The bicelles in this preparation have been confirmed by imaging in cryo-EM. (B) Mixture 
ofliposomes and open lipid films. Some parts of the films show a rippled lipid phase (arrows). The lipid films with rippled phases in this preparation have been confirmed by imaging in cryo-EM.
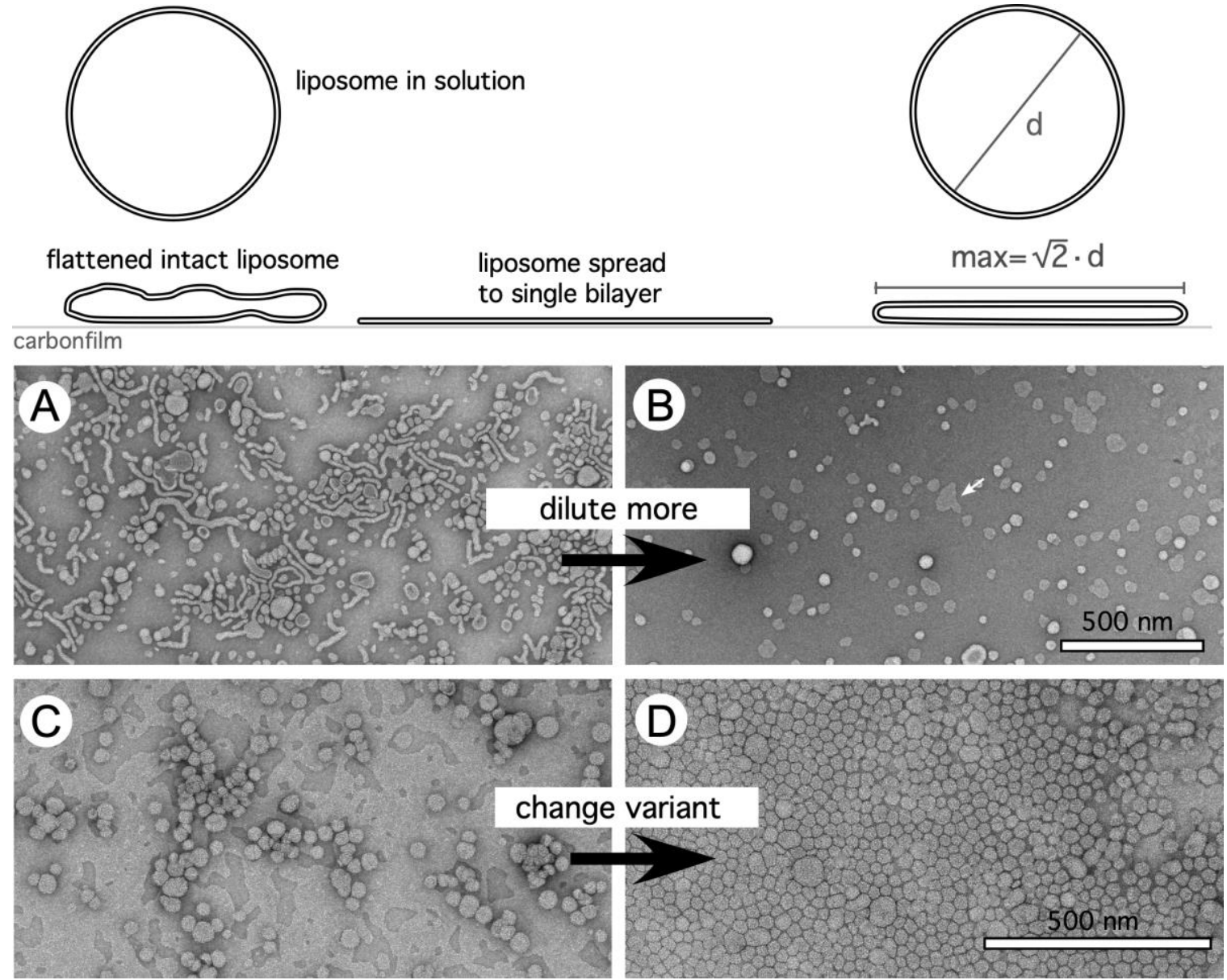

Figure 2. Interaction of liposomes with the carbon film. Schematic shows liposome in solution, intact but flattened liposome in negative stain, and a liposome spread out on the carbon film and explains the size increase of liposomes by flattening. (A) and (B) show a preparation of liposomes that tends to spread on carbon and forms worm-like micelles and tubes at high concentrations. Dilution can resolve the problem of tubes, but the liposomes are still observed to spread on the carbon film (arrow). (C) and (D) show a liposome preparation that spreads on carbon film and forms a continuous film. Using a variation of the staining protocol keeps most of the liposomes intact.

\section{References}

[1] M. Ohi et al., Biol. Proced. Online 6 (2004), p. 23-34

[2] U. Baxa, Methods Mol. Biol. 1682 (2018), p. 73-88.

[3] This project has been funded with Federal funds from the Frederick National Laboratory for Cancer Research, National Institutes of Health, under contract HHSN261200800001E. The content of this publication does not necessarily reflect the views or policies of the Department of Health and Human Services, nor does mention of trade names, commercial products or organizations imply endorsement by the US Government. 